CONGRES 92e Congrès de l'AOCS (Minneapolis, 13-16 mai 2001) : l'essentiel du programme Nutrition

Oléagineux, Corps Gras, Lipides. Volume 8, Numéro 5, 465-71, Septembre - Octobre 2001, La filière

Auteur(s) : François MENDY, 2, rue du Calvaire, 92210 Saint-Cloud, France.

Résumé : Avec plus de deux mille participants, cinq cent cinquante communications orales et posters dont quatre-vingt-onze en "Health and Nutrition ", il devient presque impossible de suivre même partiellement le programme d'autres divisions. Ce congrès, et particulièrement la Division Health and Nutrition, devait beaucoup au Secrétaire général de I'AOCS, Marc Bieber, mort malheureusement quelques jours plus tôt. Certaines des communications avaient été prévues pour amener des réponses à des questions laissées en suspens l'année précédente. Très nettement, une évolution s'amorce qui se poursuivra d'ailleurs, tant elle est la conséquence de multiples progrès. Cinq grands thèmes constituaient l'architecture du Programme de la Division " Health and Nutrition » : - nutrition générale (regroupant des communications non directement rattachables aux thèmes suivants) ; lipides sanguins et maladies cardiovasculaires ; - immunologie et inflammation ; - effets métaboliques et physiologiques des CLA ; - " sécurité " et effets santé des phytostérols ou phytostanols.

\title{
ARTICLE
}

Avec plus de deux mille participants, cinq cent cinquante communications orales et posters dont quatre-vingt-onze en "Health and Nutrition ", il devient presque impossible de suivre même partiellement le programme d'autres divisions. Ce congrès, et particulièrement la Division Health and Nutrition, devait beaucoup au Secrétaire général de I'AOCS, Marc Bieber, mort malheureusement quelques jours plus tôt. Certaines des communications avaient été prévues pour amener des réponses à des questions laissées en suspens l'année précédente. Très nettement, une évolution s'amorce qui se poursuivra d'ailleurs, tant elle est la conséquence de multiples progrès. Cinq grands thèmes constituaient l'architecture du Programme de la Division « Health and Nutrition » :

- nutrition générale (regroupant des communications non directement rattachables aux thèmes suivants) ;

- lipides sanguins et maladies cardiovasculaires ;

- immunologie et inflammation ;

- effets métaboliques et physiologiques des CLA ;

- « sécurité » et effets santé des phytostérols ou phytostanols. 
Habituellement, nous avions l'habitude de résumer brièvement l'essentiel des meilleures communications et posters en tenant compte, sinon totalement du moins essentiellement, de leurs qualités techniques. Mais, très nettement depuis cette année, un mouvement s'amorce, séparant le présent du passé. II apparaît une intrication de plus en plus grande des sujets. Dans l'avenir, tout sujet pluridisciplinaire, comme cela devient de plus en plus la règle, devra tenir compte des niveaux actualisés de chaque discipline mise en œuvre. Même si rien n'est négligeable dans un processus de mondialisation, la loi de l'homéorhésis ramenant tous les projets au plus petit commun dénominateur constituant risque de s'appliquer dangereusement à certains travaux mettant en œuvre des disciplines de niveaux de qualité trop différents.

Nous tenterons donc cette année de ne retenir que les présentations qui nous ont paru cohérentes en tous points, y compris sur le plan de la nutrition humaine, tout en sachant que ceci introduit un certain degré de subjectivité.

\section{Nutrition générale}

Douze communications et cinq posters constituaient un bel ensemble. Quatre communications nous ont paru plus importantes que les autres.

"Transformation de la testostérone (T) en dihydrotestostérone (DHT) dans les cellules prostatiques cancéreuses ou non cancéreuses : modulation de la 5 alpha-réductase par l'acide gamma-linolénique $(G L A) »$ de H.T. Pham et V.A. Ziboh, Davis, États-Unis.

Cette communication s'est vue attribuer un Honored Student Award et un Health and Nutrition Division Student Excellence Award. Le DHT est le métabolite actif de la testostérone, c'est le responsable de la plus grande production de PGE2 par les cellules cancéreuses versus les cellules non cancéreuses. Les auteurs ont testé le gamma-linolénique et son métabolite oxydatif le 15-HETrE sur la 5 alpha-réductase qui transforme la testostérone en DHT. Les résultats ont montré :

- une augmentation dose-dépendante de la conversion de T en DHT plus importante dans les cellules cancéreuses que dans les cellules normales, cela sans GLA ;

- une inhibition de $60 \%$ de la 5 alpha-réductase par le GLA à 10 mmol ;

- une inhibition de $80 \%$ par le 15 -HETrE à $10 \mathrm{mmol}$.

Ces résultats suggèrent un rôle possible des hormones testiculaires et des éicosanoïdes dans les développements/progressions du cancer de la prostate et l'atténuation des développements/progressions par du GLA endogène et/ou exogène apporté par l'alimentation.

" Acides gras et expression des cyclo-oxygénases (COX) dans des lignées cellulaires MC3T3-E1 ostéoblastes-like » de H.E. Lippman et B.A. Watkins, Food Science, Lipid Chemistry and Molecular Biology, West Lafayette, États-Unis. 
Ce travail sur l'influence des acides gras insaturés sur la COX1 (remplissant les housekeeping needs) et la COX2 (réponse inflammatoire rapide), point de départ de la synthèse des éicosanoïdes, sera confirmé et complété plus loin par le travail de J.L. Harwood. Chez l'animal du moins, un ratio faible de $n-6 / n-3$ tend à augmenter la croissance osseuse et décroît la production ex vivo de PGE2, versus un ratio élevé $n-6 / n-3$. La COX2 semble être modulée de la même manière.

Ainsi, ces deux communications ouvrent des horizons passionnants sur la régulation des hormones stéroïdiennes au niveau des tissus récepteurs, ainsi que sur des facteurs de formation des os.

Les deux autres communications que nous avons retenues portent sur la cinétique et le ciblage des acides gras insaturés. Pour un certain nombre d'auteurs, un travail qui ne tient pas compte de la position d'un acide gras saturé ou insaturé sur le triglycéride, et particulièrement de la position en sn-2 (surtout s'il s'agit d'acides gras comme le myristique ou l'alpha-linolénique, substrats privilégiés de la bêta-oxydation mitochondriale lorsqu'ils ne sont pas en sn-2) ne doit plus être retenu.

La communication "Structure régiosomérique des triglycérides dans quelques substituts communs du lait maternel " de J.P. Kurvinen, O. Sjoval et H. Kallio (Biochemistry and Food Chemistry, Turku, Finlande) pose clairement le problème de façon générale. Quoiqu'il existe de nouvelles méthodes de synthèse des triglycérides structurés, pour l'instant, on ne peut que constater de très grandes variabilités parmi les divers substituts.

Le travail de A.B. Christophe, S.R. De Vriese et M. Dhont, du département d'endocrinologie et de nutrition de l'université de Gand, intitulé "Acides gras des phospholipides et des esters de cholestérol durant la grossesse, et à l'accouchement chez la mère et le nourrisson " aborde ce problème sur le plan descriptif. Les résultats retrouvés confirment des travaux déjà anciens ( $F$. Mendy , P. Sarda et $A$. Crastes de Paulet). Le 18:0, le 20:4n-6 et le 22:6n-3 sont plus élevés chez le nouveau-né que chez la mère $(p<0,001)$, un processus de magnification biologique selon Crawford.

L'étrangeté jamais résolue de l'augmentation du palmitoléique au cours de la grossesse, au niveau des phospholipides (même in utero d'après P. Sarda), est retrouvée mais n'est toujours pas expliquée. Pour certains, elle est un signe de carence de la mère en alpha-linolénique.

Une relation linéaire est retrouvée entre le taux de 18:2n-6 dans les esters de cholestérol et les phospholipides qui rassemble les données entre la mère et le nouveau-né $(r=83, p<0,0001)$. Les auteurs concluent que la composition en acides gras des phospholipides et des esters de cholestérol change durant la gestation, obéit à des lois différentes entre la mère et l'enfant mais que, à l'approche du terme, des mécanismes semblables sont opérants pour l'incorporation des acides gras au niveau des phospholipides et des esters de cholestérol chez la mère et le fœtus. En revanche, dès l'introduction de l'allaitement maternel dans notre expérience, le pourcentage d'acide linoléique dans les esters de cholestérol cesse d'être relié au pourcentage d'acide linoléique dans le lait maternel $(r=-0,055, p=0,85)$ (F. Babin, P. Sarda, F. Mendy, B. Descomps. Eur J Clin Nut 2000 ; 54 : 840-3). 
Parmi les posters Nutrition générale, un poster remarquable est à retenir ( $2^{\mathrm{e}}$ prix des posters) : " Profils quantitatifs de la teneur en acides gras du système nerveux central de babouins prématurés et de nouveau-nés à terme, nourris à cinq régimes différents et sacrifiés à 4 semaines » de G.Y. Diau, J.T. Brenna et al., Division of Nutritional Sciences, and Laboratory of Pregancy and Newborn Research, Cornell University.

Les cinq régimes se répartissaient ainsi :

- à terme, nourris au sein $(n=5)$;

- à terme, formule sans acide gras HI (hautement insaturés) à C20-22 ( $n=5)$;

- à terme, avec des acides gras $\mathrm{HI}$ à C2O-C22 (n= 7) ;

- préterme, sans acide gras $\mathrm{HI}(\mathrm{n}=3)$;

- préterme, avec acides gras $\mathrm{HI}$ à $\mathrm{C} 20-\mathrm{C} 22(\mathrm{n}=3)$.

Après le sacrifice à 4 semaines, les cerveaux étaient disséqués en 26 régions spécifiques. Les cerveaux des animaux nourris au sein, présentaient le taux le plus élevé d'acide gras $\mathrm{HI}$ à C22:6 inclus au niveau du globus pallidus (16\%), le plus faible au niveau du nerf optique (4,5\%). En moyenne, les animaux à terme avaient $10 \%$ de moins de C22:6 au niveau du cortex cérébral, tandis que les structures centrales retenaient le C22:6. Les régimes riches en acides gras $\mathrm{HI}$ en $\mathrm{C} 20-\mathrm{C} 22$ aboutissaient à des taux similaires indépendants du statut initial de prématuré ou de nouveau-né à terme. Les groupes sans acides gras $\mathrm{HI}$ en C20-C22 avaient environ $90 \%$ du taux de C22:6 des autres. Le C22:6 était plus dépendant du régime que le C20:4. Les structures à base de myéline avaient davantage de saturés que d'acides gras $\mathrm{HI}$ en $\mathrm{C} 20$ et $\mathrm{C} 22$. Ce travail constitue une mine extraordinaire de renseignements. Une analyse détaillée des tableaux faisait apparaître dans certaines régions des taux élevés de C22:4n-6, ou acide adrénique, le troisième acide gras hautement insaturé du cerveau et dont pourtant on ne parle pratiquement jamais. Depuis le travail initial de O'Brien J.S. et Lois Sampson E. (J Lipid Res 1965 : 537-550) sur des cerveaux de nourrissons post-mortem, aucun travail de cette ampleur n'avait été réalisé. Sa parution in extenso rendrait de très grands services.

Un travail un peu provocant, "Le mystère de l'acide docosapentaénoïque : de l'acide d'Osbond à mon éventuelle déficience peroxysomale " de R.G. Ackman, Halifax, Canada, soulève le problème du C22:5n-3 et des confusions, tout aussi fréquentes sinon plus qu'il y a 30 ans, avec le C22:5n-6. Et, curieusement, il introduit une confusion d'analyste cette fois. II y a en fait un problème non suffisamment élucidé des rôles respectifs du C22:4n-6 ou acide adrénique et du C22:5n-3. Ce sont ces deux acides gras qu'il faut comparer. $\mathrm{Si}$, dans des situations de carence en $\mathrm{n}-3$, le C22:5n-6 remplace plus ou moins, pour certains rôles, le C22:6n-3 ou DHA (double liaison en delta 4), pour les physiologistes, c'est le C22:4n-6 (double liaison en delta 7) qui joue un rôle probablement très important et qui, en phase de carence en $n-6$, est plus ou moins remplaçable par le C22:5n-3 (double liaison en delta 7). 
Enfin, in vivo (dans le cadre de chemins préférentiels), le C22:4n-6 est une réserve, une source endogène de C20:4n-6 dont le taux en tant que précurseur d'éicosanoïdes doit être sévèrement contrôlé. En revanche, il ne semble pas que le C22:5n-3 agisse de la même façon dans le lait maternel : c'est une réserve exogène. Le C22:6n-3 ne dispose pas d'« une voie de garage en attente " comme l'est le C22:4n-6 pour le C20:4n-6.

Toujours dans cette recherche de chemins préférentiels de systèmes de régulation, le groupe de $\mathrm{AJ}$ Sinclair a présenté "Une cinétique d'incorporation du 1-14C alpha-linolénique dans des tissus variés chez le rat » de N. Attar-Bashi et A.J. Sinclair, RMIT University, Melbourne, Australie. Si intéressants que soient certains des résultats, l'alpha-linolénique, acide gras libre, même instillé en intrapéritonéal, n'a pas grand chose à voir avec un alpha-linolénique fixé en sn-2 sur une molécule de triglycéride qui, d'emblée, entre par un chemin préférentiel.

\section{Lipides sanguins et maladies cardiovasculaires}

Une session analogue sur ce thème avait eu lieu au $91^{\mathrm{e}}$ Congrès de l'AOCS à San Diego en avril 2000 (OCL $2000 ; 4$ : 388-98). La question posée "Acides gras mono-insaturés versus polyinsaturés dans la gestion du risque cardiovasculaire : que devrions-nous choisir ? " était restée presque sans réponse. L.L. Rudel avait lourdement insisté sur le danger des rapports oléique/linoléique trop élevés au niveau des apports alimentaires, sur le danger de la trop grande sécrétion hépatique d'oléate de cholestérol dans les lipoprotéines contenant de l'apoB : " This hepatic secretion rate was proportional to the extent of coronary artery atherosclerosis... activation of ACAT2 may be the reason dietary monounsaturated fat promotes atherosclerosis out of proportion to its apparent by-effects on plasma LDL and HDL cholesterol concentration. "P. Reaven avait insisté sur l'oxydation des lipoprotéines et l'induction de réponses pro-inflammatoires par les acides gras polyinsaturés en excès. Et Marc Bieber avait conclu que, pour une industrie fondée sur le traitement des matières grasses, la réponse qui apparaissait « ni saturés, ni polyinsaturés, ni mono-insaturés » ne pouvait être acceptable. Seul l'acide alpha-linolénique était épargné. J'avais prévenu Marc Bieber que nous pourrions peut-être apporter une autre réponse en 2001.

La session 2001 comportait six communications et quatre posters.

\section{Influence de la forme d'apport des acides gras sur des données physiologiques chez l'homme}

Trois communications concernaient ce sujet.

"Absorption et métabolisme des triacylglycérols structurés chez l'homme " présentée par le groupe de C.E. Hoy, Biocentrum, Technical University of Denmark.

La conclusion confirme d'autres travaux précédents. L'importance de la position en sn-2 se vérifie de plus en plus : " the results indicate that diet which includes structured triglycerides may be used to direct fatty acids toward hepatic oxydation or extrahepatic uptake ».

" Effet à long terme de diacylglycérols diététiques sur les lipides sériques et le métabolisme du tissu adipeux chez l'homme » de K. Yasunaga, T. Matsuyama et al., Corporation, Health Care, Products Res. lab., Tokyo Metropolitan Nat. Health Ins., Department Pediatrics, Tokyo. 
Quatre garçons et neuf filles de 7 à 17 ans, soignés en consultation pour hyperlipémie et obésité, ont été mis pendant cinq mois sous ce produit utilisé comme huile de cuisson. La graisse abdominale mesuré par CT-Scan au niveau ombilical diminuait significativement, alors que le BMI ne changeait pas. Sur le plan sanguin, le taux de leptine baissait, les triglycérides baissaient et le C-HDL augmentait significativement dans la dernière partie de l'étude.

"Effets des diacylglycérols diététiques sur la réduction de la lipémie post-prandiale, le poids corporel et le tissu adipeux » de N. Matsuo, K.C. Maki, N. Tada et al., Kao Corporation, Japon ; Chicago Center for Clinical Research ; Jikey Univ. School of Medecine, Japan.

Un DAG (70 \% en 1,3-isoforme) et un TAG de contrôle amenant les mêmes acides gras étaient utilisés. Deux études étaient mises en place : une en aigu, une au long cours.

Étude en aigu : doses de $30 \mathrm{~g}$ de lipides par $\mathrm{m}^{2}$ de surface corporelle ( $\mathrm{n}=6$ hommes). Les aires sous la courbe des TG sériques, des TG des remnants et du Cholestérol des remnants après une surcharge en DAG diminuaient de 12,9\%, 28,3\%, 17,3\%. Les lipides des remnants reflétaient mieux les différences que les lipides totaux sériques.

Étude sur un long terme : 65 hommes et femmes obèses ou en surpoids étaient choisis et répartis au hasard en deux groupes, un sous DAG, l'autre sous TAG durant 24 semaines.

L'huile testée représentait $15 \%$ de l'énergie totale d'une diète hypocalorique (500 à 800 Cal de déficit). Les sujets sous DAG maigrissaient de façon significativement plus efficace. L'huile à base de DAG est autorisée au Japon comme FSHU depuis mai 1998.

La quatrième communication, "Effet d'une huile de poisson et d'un supplément de levure rouge de riz seul ou en combinaison sur des facteurs de risque cardiovasculaire connus " de A. Frazer et B. Holub, Univ. de Guelph, Dpt of Human Biology and Nutritional Science, Canada, était très intéressante à deux points de vue. Si l'effet de l'alpha-linolénique est définitivement validé par l'American Heart Association, les effets des huiles de poisson sont documentés mais ses inconvénients commencent à paraître et B. Holub avait jusqu'ici beaucoup de mal à l'accepter. Dans ce travail, B. Holub reconnaît que, si les huiles de poisson abaissent les TG, souvent ils n'améliorent pas le cholestérol-LDL et parfois même l'augmentent. D'où l'idée d'ajouter un extrait de levure rouge de riz contenant un inhibiteur naturel de I'HMG-coA réductase ; 50 sujets mâles hypercholestérolémiques ont été ainsi répartis en quatre groupes au hasard:

- placebo ;

$-2,4 \mathrm{~g}$ d'extrait de levure rouge de riz ;

- 6 g d'huile de poisson (3 g AGHI en $n-3$ );

$-2,4 \mathrm{~g}$ d'extrait de levure de riz $+6 \mathrm{~g}$ d'huile de poisson ( $3 \mathrm{~g}$ AGHI en $n-3)$.

À la $8^{\mathrm{e}}$ semaine, les meilleurs résultats étaient obtenus avec le mélange huile de poisson + extrait de levure rouge de riz. Chol-T : - 20 \%, C-LDL : - 22,7 \%, TG : - 39,2\%, les rapports CT/C-HDL, C-LDL/CHDL, TG/C-HDL étaient aussi significativement améliorés. 
Si l'on compare ces résultats à des études au long cours utilisant seulement chez l'homme $1 \%$ de l'E (énergie totale) sous forme d'acide alpha-linolénique en sn-2, soit 2,5 à $3 \mathrm{~g}$, il s'avère que les résultats obtenus avec $3 \mathrm{~g}$ d'EPA et de DHA + 2,4 g d'extrait de levure rouge de riz sont plutôt moins bons.

\section{Les travaux « French Team »}

Les deux autres communications orales et les trois posters correspondants ont été regroupés par $M$. Craig-Schmidt sous le nom de travaux de la « French Team » et le $1^{\mathrm{er}}$ prix des posters a été attribué à I'un des posters. II faut admettre que la validation de la Lyon Diet Heart Study de S. Renaud et M. de Lorgeril en juillet 2000 par l'American Heart Association Science Advisory and Coordinating Commitee (après six ans de réflexion) a donné une certaine crédibilité aux travaux français en ce domaine. Le rapport a maintenant été publié (Circulation 2001 ; 103 : 1823-5, P. Kris-Etherton et al.). II souligne en particulier combien " an independant role is assigned to the activity of alpha-linolenic acid in lowering cardiovascular disease... a modified AHA step 1 diet be introduced to take advantage... in the population at large. The components of the modified Step 1 diet should include... foods high in alpha-linolenate... flaxseed ail, canola oil and non-hydrogenated vegetable oil salad dressing. The provision of the desirable monoinsaturated fatty acid can be achieved through the use... of canola oil ».

Les cinq travaux exposés comportaient tous $1 \%$ de l'énergie totale sous forme d'acide alphalinolénique dans leur régime et $5 \%$ sous forme d'acide linoléique. Ces deux points de base figés, une première série s'intéressait au pourcentage d'acide oléique souhaitable pour répondre aux questions posés par L.L. Rudel à la session de l'AOCS sur ce sujet en 2000 et par S.M. Grundy (Am F Clin Nutr, 1997 : S988-90).

"Réponse lipidique post-prandiale retardée. Distribution apo CIII et capacité d'efflux du cholestérol du plasma chez des sujets normolipidémiques avec ou sans maladie coronarienne prématurée » de B. Delplanque, B. Le Roy, C. Senault, F. Sueda, A. Thaminy et F. Mendy, Univ. Paris-Sud, Univ. de Rennes, Fac de Médecine X Bichat Paris, Cetiom et Onidol.

"Réponse triglycéridique post-prandiale, insulinémie et distribution apo CIIl chez des sujets humains normolipidémiques » de B. Le Roy, B. Delplanque, F. Mendy, D. Gripois, M.F. Blouquit, A Thaminy, F. Syeda et E. Fenart, Univ. Rennes, Univ. Paris-Sud, Cetiom et Onidol, et les moines de I'Abbaye de Solesmes, présentation à laquelle a été décernée le $1^{\text {er }}$ prix des posters.

" Acide alpha-linolénique dans les esters de cholestérol plasmatiques : un marqueur de l'apport d'acide alpha-linolénique chez les humains " de N. Combes, B. Delplanque, S. Tanguy, C. Boue, F. Mendy, B. Le Roy et E. Fenart, Iterg-Biochimie-Nutrition, Univ. Bordeaux-1, Univ. Paris-Sud, Univ. Rennes et Cetiom et Onidol, et les moines de l'Abbaye de Solesmes.

"Acides oléique, linoléique et alpha-linolénique amenés par les huiles végétales : quels sont les limites des effets bénéfiques sur la lipémie et sur les paramètres athéro-thrombotiques chez les humains ? „de B. Delplanque, B. Le Roy, F. Mendy, E. Fenart, A. Thaminy, F. Syeda, N. Combe et A. Ruelland, Univ. Paris-Sud, Univ. Rennes, Iterg-Biochimie-Nutrition, Univ. Bordeaux-1, Cetiom et Onidol, et les moines de l'Abbaye de Solesmes. L'objectif de cette étude était de définir les ratios souhaitables OL/LA/ALA et de valider les niveaux diététiques optima d'acide oléique (OL) après 
stabilisation du rapport LA/ALA dans le régime de 34 sujets mâles. Trois niveaux d'acide oléique (11, 13 et $16 \%)$ ont été testés sur des périodes de quatre mois et les clairances lipidiques post-prandiales étaient testées après un repas d'épreuve à la fin des quatre mois.

Après une série impressionnante d'analyses, il apparaît que l'on peut proposer comme marge de sécurité pour une population normale d'adultes " dans le cadre d'un régime à $2000-2500 \mathrm{cal} / \mathrm{j}$ et dans le cadre d'un apport en linoléique de 4 à $6 \%$ de l'énergie (9 à $13 \mathrm{~g} / \mathrm{j}$ ) et en alpha-linolénique de $1 \%$ de l'énergie $(1,5$ à $2,5 \mathrm{~g} / \mathrm{j})$ ", un apport en oléique de 11 à $16 \%$ de l'énergie (soit 28 à $47 \mathrm{~g} / \mathrm{j}$ ). Enfin, le niveau de $13 \%$ d'énergie sous forme d'oléique semble représenter un petit avantage supplémentaire.

Très intéressés par ce poster, les organisateurs du congrès AOCS Amérique du Sud ont demandé l'autorisation de l'emporter, pour le présenter au cours de ce congrès.

La $2^{\mathrm{e}}$ communication orale de la " French Team " "Effets de trois doses croissantes de myristique en sn-2 (0,6-1,2-1,8 des calories totales) sur des paramètres lipidiques dans une collectivité de moines " de H. Dabadie, E. Peuchant, M. Bernard, M.F. Dumon, L. Lalande, N. Combe, H. Gin, P. Le Ruyet et F. Mendy, hôpital Haut-Lévêque Pessac, hôpital St-André Bordeaux, Iterg-Biochimie-Nutrition, Univ. Bordeaux-1 Talence, Lactalis, Rétiers, France et les moines de l'Abbaye de Bellocq, soulevait un autre problème toujours évité. " II ne suffit pas d'amener dans l'alimentation, en doses et rapports convenables, les trois acides gras parents ou précurseurs, oléique, linoléique, alpha-linolénique en sn-2, il faut de plus réunir les conditions métaboliques favorisant leur transformation en dérivés supérieurs par des chemins préférentiels biologiquement contrôlés (en général cinq étapes de contrôle minimum)".

Cette attitude semble plus éthique que d'ajouter ces mêmes dérivés supérieurs sous forme d'acides gras libres, ou présents sur des triglycérides à des positions inadéquates ou incertaines.

Dans un régime de $2300 \mathrm{cal} / \mathrm{j}$, comprenant en pourcentage de l'énergie totale $11 \%$ d'oléique, $5 \%$ de linoléique, 0,9\% d'alpha-linolénique chez 16 moines sans dyslipidémies, par périodes successives de cinq semaines suivies d'un wash-out, ont été introduits $0,6 \%$ de myristique en sn-2 (lait $1 / 2$ écrémé), $1,2 \%$ (lait entier), 1,8 \% en sn-2 grace à un mélange d'huile de beurre fractionnée appauvrie en stéarique, palmitique, rapport myristique/palmitique, ou myristique/stéarique profondément modifié.

Ces apports « physiologiques » en myristique en sn-2 (égal à $60 \%$ de celui du lait maternel pour le lot $1,8 \%$ E) ont entraîné chez ces moines adultes, dans le cadre de ce régime, une amélioration des paramètres lipidiques. L'effet du lot 1,8 , sous forme de myristique, est supérieur significativement à celui des lots $0,6 \%$ et $1,2 \%$, évoquant l'existence d'une courbe en $U$.

Ce travail a suscité un vif intérêt chez le $\operatorname{Pr}$ A. Sinclair de Melbourne, past-president de la Division Health and Nutrition, qui a souhaité recevoir le maximum de renseignements possibles.

L'ensemble des données amenées par la « French Team » est considérable et difficile à résumer en quelques mots. II mériterait de faire l'objet d'un dossier. 


\section{Immunologie et inflammation}

Le rôle des phénomènes inflammatoires dans le développement de l'athérosclérose est de plus en plus reconnu. Le rôle des phénomènes inflammatoires chroniques dans la morbi-mortalité des personnes âgées s'avère de plus en plus important. La session " Immunologie et Inflammation » est ainsi appelée à devenir l'une des plus importantes des sessions animées par la Division Health and Nutrition de I'AOCS.

Des neuf communications programmées nous en retiendrons particulièrement trois.

"Lipides diététiques et fonction immunitaire " de J.J. Turek, Dept of Basic Medical Science Purdue University, West Lafayette. On pense habituellement que les lipides alimentaires et particulièrement ceux en n-3 agissent en modulant les synthèses d'éicosanoïdes produits par la voie des cyclooxygénases ou des lipo-oxygénases. Ceci est retrouvé dans toutes les espèces étudiées. Cependant, d'autres effets sur la production de cytokines et la fonction lymphocytaire sont très différents d'une espèce à l'autre. Ainsi, la production de Tumor Necrosis Factor est réduite chez l'homme par les n-3, augmentée chez les rongeurs, non modifiée chez le chien. Peut-être ceci vient-il de différences de susceptibilité à l'oxydation des acides gras en n-3 selon les espèces, ou à l'intérieur même d'une espèce. Or, les acides gras en n-3, particulièrement ceux hautement insaturés et à très longue chaîne, sont très sensibles à l'oxydation.

"Effets métaboliques anticancéreux et anti-inflammatoires de l'acide stéaridonique (SDA) " de A. Kabaki, J. Whelan, M.F. McEntée, V. Ursin et al., Pharmacia Corp, St-Louis, Dpt Nutrition, Univ. de Tennessee, Calgène Davis. Les auteurs ont étudié l'effet de ALA, C18:4n-3, EPA, DHA dans divers modèles de pharmacologie ayant trait à l'inflammation chez le rat. Les acides gras étaient donnés sous forme d'esters éthyliques. L'effet du C18:4n-3 est particulièrement intéressant, supérieur à I'EPA, égal à celui du DHA. La production de SDA (C18:4n-3) comme " pro-EPA/DHA » par une plante végétale pourrait s'avérer intéressante. Calgène a pu ainsi obtenir un colza génétiquement modifié à $25 \%$ de linolénique et $25 \%$ de SDA.

La $3^{\mathrm{e}}$ communication pourrait par ses retombées, si elle était confirmée, devenir la plus importante acquisition de ce congrès. "Les acides gras polyinsaturés en $n-3$ inhibent les réponses inflammatoires dans un système de modèle d'arthrite expérimentale " de J.L. Harwood, C.L. Curtis (Honored student award winner), S. Hurst, C.E. Hughes, C.R. Flannery, C.B. Little et B. Caterson, Cardiff Univ. School of Biosciences, Cardiff, UK.

La composition en acides gras des membranes de chondrocytes était modifiée grâce à l'addition de différents acides gras complexés à l'albumine, ajoutés au milieu de culture. L'alpha-linolénique, I'EPA, le DHA, le palmitique, l'oléique, le linoléique, l'arachidonique étaient ainsi ajoutés alternativement. Seuls les acides gras en $n-3$ réduisent de façon dose-dépendante l'expression de I'ARNm de la cyclooxygénase 2 inductible, alors qu'ils n'ont pas d'effet sur l'expression de la cyclo-oxygénase 1 constitutive. Seuls les acides gras en n-3 suppriment l'expression de la 5-lipoxygénase, mais ils auraient peu d'effets sur la 12-lipoxygénase. Enfin seuls les acides gras en n-3 diminuent les cytokines inflammatoires et les protéinases dégradant les cartilages. Si ceci était confirmé chez I'homme, l'importance de cette découverte serait très grande. 


\section{Effets métaboliques et physiologiques des CLA (Conjugated Linoleic Acid)}

Quinze communications orales, quatre posters : c'était en principe la session la plus importante animée par la Health and Nutrition Division. Pourtant, la réflexion ironique qui venait en mémoire était celle de J.C. Stanley de Lincoln Edge Nutrition à un symposium analogue organisé par la SCI (Society of Chemical Industry) à Londres le 15 mars 2001 : "Similar-sounding claims were made for dietary fiber in the 1970s!"

Les CLA sont un mélange de six isomères dont l'un est naturel, présent dans tous les laits de mammifères y compris le lait de femme : I'acide ruménique ou C18:2n-7trans, 9cis,11trans(figure 1, linoléique, versus figure 2, ruménique, et figure 3, isomère 10trans, 12cis CLA) et cinq, non naturels, sont des molécules étrangères à la biologie humaine. L'acide ruménique est présent à des taux de 4 à $5 \mathrm{mg} / \mathrm{g}$ de lipides dans la viande d'agneau, de bœuf, parfois plus dans les produits laitiers et peut atteindre le double dans le lait maternel.

Traditionnellement, les cinq autres représentaient autour de $0,7 \mathrm{mg} / \mathrm{g}$ dans les huiles végétales et étaient en principe formés au cours de la désodorisation et des processus de raffinage. La production du mélange de six isomères par isomérisation alcaline de l'acide linoléique est aisée. L'isolement de l'acide ruménique est coûteux, de même que sa synthèse.

Produit naturel, l'acide ruménique n'est pas brevetable. Mais si, par hasard, une synergie apparaissait entre l'acide ruménique et l'un des autres isomères le produit le deviendrait, d'où tous les brevets pris par W. Pariza. Nous avons vu ainsi apparaître des produits contenant les six isomères, puis quatre, enfin deux : I'acide ruménique 9cis,11trans et le 10trans,12cis (figure 3) en un mélange $50 / 50$.

Ce sujet devenait enfin une manne financière pour les spécialistes de l'analyse chimique des lipides, permettant le renouvellement de tout un matériel et l'acquisition de techniques nouvelles. D'une modeste HPLC en phase inverse, on est passé à une HPLC en phase liquide (ion-argent), puis à des systèmes de six colonnes en phase solide montées en série (Yurawecz, FDA, Washington) et à des chromatographies en phase gazeuse couplées avec une spectrométrie de masse. Cela ne disposait guère à développer un sens critique aigu.

Or, une analyse approfondie sur le plan chimie structurale, biochimie comparée, chimie physiologique, physiologie humaine, réglementation pharmaceutique, aboutissait, elle, à un résultat inverse.

Des six isomères, seul l'acide ruménique 9cis,11trans,n-7trans, seul isomère naturel, partage avec l'acide linolénique (n-3), l'acide linoléique (n-6), l'acide oléique (n-9), trois caractéristiques liées entre elles, privilèges uniques de ces quatre acides gras :

- 18 atomes de carbone ;

- une $1^{\text {re }}$ double liaison cis en delta 9 ;

- la possibilité d'être le point de départ d'une famille en n-3, n-6, n-7trans, n-9. 
In vivo, l'acide ruménique dispose d'une " réserve biochimique ", d'un acide gras "parent ", l'acide vaccénique C18:1n7trans. Sur le plan chimie structurale, le " ruménique » est situé entre l'oléique, le linoléique, le linolénique dans un gradient continu sur plusieurs points. Sur Je plan physiologique, au niveau du lait humain, son apport est légèrement supérieur ou égal selon les circonstances à celui du linolénique. Mais si, ce qui est fort probable, cela se confirmait brusquement, le problème de la législation sur les trans serait remis en cause. II apparaît nécessaire de séparer les trans d'origine industrielle des trans d'origine biologique que sont le "vaccénique " et le "ruménique ", parfaitement définis sur le plan biochimique et jouant sans doute des rôles essentiels. C'est dans cet esprit, et l'esprit de notre introduction que nous n'avons retenu que trois communications et un poster.

"Différences dans l'incorporation de CLA dans différents tissus, et dans diverses classes lipidiques chez des porcs consommant soit un mélange de deux soit de quatre isomères »de J.K.G. Kramer, J. Zhou, J. Zhang, M.E.R. Dugan et J.L. Aalhus, Agriculture and Agri-Food Canada, Food Research Program, Guelph, et Agriculture and Agri-Food Canada, Lacombe, Alberta, Canada.

Le mélange à quatre isomères était donné au taux de $2 \%$ du régime de base, vs $2 \%$ de tournesol. Le mélange à deux isomères était donné sous la forme de $0,5 \%$ dans $5 \%$ de Canola. Des quatre isomères le 9c, 11tr et le 11c,13tr étaient les mieux incorporés. À partir du mélange de deux isomères, le $9 c, 11$ tr est incorporé de façon prédominante dans toutes les classes lipidiques, sauf dans les triglycérides ou la teneur en les deux isomères reflète l'apport. Les résultats suggèrent que ces mélanges d'isomères inhibent plutôt le métabolisme des acides gras en $n-6$, et activent le métabolisme des acides gras en n-3.

Les CLA préservent le rythme de formation des os chez le rat en croissance. Y. Li, M.F. Seifert, B.A. Watkins, Purdue Univ. West Lafayette, School of Medicine, IUPUI, Indianapolis.

Dans cette étude réalisée sur 80 rats, répartis en quatre groupes suivant la teneur du régime en huile de soja, en graisse de bœuf, avec 0,5 \% de CLA ou non, les CLA abaissaient le C18:1n-9, le palmitoléique, tandis qu'ils augmentaient le C22:6n-3 et le total des acides gras en n-3 dans la fraction polaire de la moelle osseuse. Le taux d'arachidonique, dans cette même fraction n'était pas modifié, mais il était très nettement abaissé dans les lipides neutres des autres tissus. À 12 semaines, le taux d'ostéocalcine sérique, les phosphatases alcalines sériques étaient abaissés. À la fin de six semaines de régime, la formation d'os endostéale tibiale était augmentée pour le lot consommant 25 $\%$ de graisse de bœuf $+0,5 \%$ de CLA (et 74,5\% d'huile de soja). À la fin de 12 semaines, ce résultat était maintenu. II semble que le niveau d'apport de CLA soit un facteur jouant sur la régulation du métabolisme osseux chez l'animal en croissance.

Un poster : "Taux plasmatique et teneur du lait maternel en CLA, durant la grossesse et la lactation ", C.J. Lammi-Keefe, S.Y. Lee, V. Wijendran, Univ. Connecticut, U-17, Storrs, USA, a confirmé la relation existant entre l'apport alimentaire en CLA et la teneur en CLA du plasma et du lait maternel. L'apport variait de $33,2 \mathrm{mg} / \mathrm{j}$ à $261 \mathrm{mg} / \mathrm{j}$, avec une moyenne à $131,6 \mathrm{mg} / \mathrm{j}$. Dans cette population, la source principale de CLA étant les produits laitiers, suivis par le bœuf, l'isomère principal présent dans les ingestats était donc l'acide rumérique, 9cis, 11trans 18:2. 
L'isomère principal retrouvé dans le plasma et le lait maternel est le ruménique, avec un peu de 9trans, 11trans 18:2. Pratiquement aucun autre isomère n'est retrouvé. L'apport alimentaire en acide ruménique est donc un déterminant du taux de ruménique du lait maternel.

Mais en ce domaine, et d'ailleurs de façon générale, l'un des éléments marquant du Congrès a été la communication de H.M. Roche, E. Noone, A. Nugent, M.J. Gibney, Saint-James Hospital, Ireland : " Nutrient Regulation of SRBEP1C Gene Expression : CLA reduces plasma TAG concentrations by down regulating hepatic SREBP1C in an isomer-specific effect of CLA on lipoprotein metabolism in man and gene expression in $\mathrm{Ob} / \mathrm{Ob}$ Mice »

24 souris mâles Ob/Ob étaient réparties au hasard pour recevoir un régime amenant soit du 9cis, 11trans C18:2 ruménique), soit du 10trans, 12cis C18:2, soit du linoléique (régime contrôle) pendant quatre semaines. Le 10trans, 12 cis C18:2 réduit significativement le poids corporel, et l'adiposité ( $p<$ $0,0001)$, mais affecte de façon négative les triglycérides plasmatiques, les acides gras non estérifiés, le glucose, et le métabolisme de l'insuline. Le ruménique réduit significativement les TG plasmatiques $(p<0,01)$, et les acides gras non estérifiés $(p<0,05)$. Cela était associé à une " down-regulation " significative de I'Hepatic Sterol Regulatory Response Element Binding Protein 1c (SREBP 1c) ( $p<$ $0,005$ ) et de l'Hepatic Tumor Necrosis Factor (TNF alpha) ( $<0,008)$. SREBP $1 c$ est un médiateur clé de la régulation nutritionnelle de la transcription des gènes impliqués dans la synthèse hépatique des acides gras et des triglycérides. Les auteurs proposent ainsi que le 9cis,11trans réduit les taux de triglycérides et d'acides gras non estérifiés en réduisant l'expression du TNF alpha, qui inhibe l'activité SREBP 1c et, de là, réduit la synthèse des acides gras et des triglycérides.

Cette étude a été ensuite poursuivie par une étude, chez 51 hommes normolipidémiques, du métabolisme des triglycerids rich lipoprotein et du transport reverse du cholestérol. Les sujets étaient répartis au hasard, pour recevoir $3 \mathrm{~g} / \mathrm{j}$ d'un mélange 50/50 de 9cis,11trans et 10trans,12cis ou d'un mélange $80 / 20$ de 9 cis-11trans et 10trans,12cis. Le mélange 50/50 de CLA réduisait significativement $(p<0,005)$ le taux à jeun de TG plasmatiques.

Le mélange $80 / 20$ réduisait significativement $(p<0,05)$ le taux de VLDL plasmatiques, mais il n'y avait pas d'effet sur le C-LDL, le C-HDL ou le métabolisme inverse du cholestérol. Le cis9-11trans était incorporé dans les lipides plasmatiques.

\section{Sécurité et effets santé des phytostérols et phytostanols}

Onze communications orales et un poster constituaient le programme de ce symposium sur un sujet d'actualité.

Quatre communications venaient d'Europe, deux sur les phytostérols du groupe Unilever et deux sur les phytostanols du groupe du Département de biologie humaine de Maastricht (R.P. Mensik et J. Plat). Ces travaux ont déjà été suffisamment vulgarisés en Europe pour qu'il ne soit pas nécessaire d'y revenir mais il peut être intéressant de relever que, avec les critères retenus, il n'y a pas chez I'homme de différences entre phytostérols et phytostanols.

De même, les études extensives de toxicologie de P. Hepburn sur le rat n'ont relevé aucune anomalie et « in recognition of this, phytosterols-esters have been affirmed as gras ». 
Ces résultats ont été confirmés au Japon chez des sujets moyennement hypercholestérolémiques par M. Sugano et al. (Univ. de Kumamoto, School of Medicine de Tokyo).

Une dose de $2 \mathrm{~g} / \mathrm{j}$ de phytostanols serait suffisante pour les Japonais, $2,5 \mathrm{~g} / \mathrm{j}$ de phytostanols pour les Hollandais, et $2 \mathrm{~g} / \mathrm{j}$ de phytostérols.

Trois autres études chez l'animal amènent des points de vue légèrement différents.

À la suite d'une étude chez des rats Sprague-Dawley $(n=25)$, le groupe d'A. Sinclair à Melbourne reste relativement neutre. Cette étude a utilisé un rapport phytostérols (extrait de l'huile de soja) sur huile de carthame dans le régime allant de 0 à 1, 2, 3, $4 \mathrm{~g}$ pour $70 \mathrm{~g}$ d'huile. Il apparaît une plus haute incorporation de ces phytostérols au niveau du cœur, des érythrocytes, du poumon et de la rate (teneur multipliée par trois) que des autres tissus $(p<0,05)$.

L'étude de I. Ikeda et al. (Univ. de Kyushu, Fukuoka, Univ. de Kumamoto) fait remarquer de façon aussi neutre que l'absorption de phytostérols est plus élevée chez les rats SHRSP (rats spontanément hypertensifs présentant des accidents vasculaires cérébraux) que chez des rats W (Wistar king A) pris comme contrôle. Les rats SHRSP ont une absorption intestinale plus élevée et une excrétion biliaire diminuée.

C'est à partir d'études sur cette race de rats SHRSP qu'une équipe canadienne présente des réflexions un peu différentes dans son étude : "Adverse health effects of plant sterols and stanols on SHRSP and diabetes-prone rats: the other side of the story " de W.M.N. Ratnayake et M.R L'Abbe (Health Canada Nutrition Res division, Health products and Food branch, Banting Res. Cent, Ottawa, Canada). Les auteurs reconnaissent qu'une augmentation de 10 fois la consommation journalière de phytostérols permet de baisser de 10 à $15 \%$ le taux de cholestérol-LDL chez des sujets moyennement à modérément hypercholestérolémiques et que, jusqu'ici, aucune étude chez des hommes sains et des animaux de laboratoire n'a montré d'effets adverses. Cependant, les phytostérols et les phytostanols réduisent l'espérance de vie des rats SHRSP d'environ 10 à $15 \%$, les phystostanols réduisant l'espérance de vie de façon plus nette que les phytostérols. Cela est associé à une plus large incorporation des phytostérols et des phytostanols dans les membranes érythrocytaires, à une réduction du nombre des plaquettes et à une diminution de la déformabilité érythrocytaire (rendant le passage plus difficile au niveau des petits vaisseaux). Une étude préliminaire confirme ce phénomène chez une souche de rats diabetes-prone.

Phytostérols et phytostanols étant reconnus GRAS, on attendait la publication de la prise de position du Comité scientifique et de coordination de I'American Heart Association. Prise en novembre dernier, elle vient d'être publiée dans Circulation, $2001 ; 103: 1177-9:$ « ... these products should be reserved for adults requiring lowering of total and LDL cholesterol levels because of hypercholesterolemia or the need for secondary prevention after an atherosclerotic event ".En fait, bien que le Comité reconnaisse " ... promising addition to dietary interventions, aimed at improving cardiac risk profiles ... ", il demande que des informations complémentaires soient reunies "... before their routine ingestion is recommended in the general population ". 


\section{Conclusion : hier, aujourd'hui, demain}

Dans le contexte précisé dans notre introduction, nous avons choisi de nous concentrer, au cours de ce $92^{\mathrm{e}}$ congrès de l'AOCS, sur ce qui concernait aujourd'hui et demain matin, mais l'un des mérites de ces congrès de l'AOCS est de savoir se souvenir d'avant-hier et d'ouvrir le chemin de l'après-demain. Ce sera notre conclusion.

Ralph T. Holman a reçu cette année le AR Baldwin Distinguished Service Award, le prix le plus prestigieux de l'AOCS et, à ce titre, a présenté sa conférence au cours du dîner de la Health and Nutrition Division. De par « sa filiation » directe avec G. Burr qu'il réunit en 1980 avec Ulf von Euler au cours d'un congrès à Minneapolis, il représente avant-hier. C'était sa $55^{\mathrm{e}}$ année de membre de I'AOCS. Avec J.F. Mead, décédé il y a quelques années, ce furent les deux grands pionniers de 1945 à 1970. Certains se sont sentis plus proches de J.F. Mead et il semble bien que ce soit cette filiation qui enjambe le $3^{\mathrm{e}}$ millénaire.

Mais, après une période d'intense production entre 1956 et 1964 autour du linoléique, brusquement, en 1982, R.T. Holman amenait la preuve formelle (Am J Clin Nutr, 1982 ; 35 : 617-23) que l'alpha linolénique était indispensable et essentiel chez l'homme et que ce dernier était capable de le transformer en dérivés supérieurs, EPA et DHA. Les preuves amenées étaient d'ordre biochimique et clinique. Une page était ouverte, qui vient de se conclure par la validation par l'American Heart Association de la Lyon Diet Heart Study.

Pourtant, le numéro suivant de l'Am J Clin Nutr amenait un article de S. Carlson suggérant que seuls I'EPA et le DHA (huiles de poisson) étaient utiles, l'alpha-linolénique n'étant pas transformé par I'homme. Un parfum de "Start-up » et de starisation l'emportait, au mépris des connaissances acquises. Sans doute dix ans ont-ils été perdus pour certains suiveurs. Il aura fallu la deuxième vague des travaux de H. Sprecher (OCL, $1997 ; 7$, AOCS $91^{\mathrm{e}}$ congrès, Supelco/Nicholas Pelick Res. Aw. de H. Sprecher) en 1992 pour recentrer le débat dans le domaine scientifique.

Mais ces travaux de H. Sprecher, A. Spectror, W. Lands et E. Emken, dans la tradition de J.F. Mead, ont relancé une grande tradition de rigueur.

Ainsi, deux exposés au cours du mini-symposium sur "Phospholipides dans le domaine de la Santé et de la Nutrition wouvraient la voie à demain et après-demain. Celui de N. Salem Jr (Lab. des Membranes: Biochimie-Biophysique, NIAA, NIH) et celui de J.T. Brenna et al. (Cornell Univ, Tajen Tech. Inst. Taiwan, Numico Res Gr. Friedrichdorf, Germany).

Les progrès récents des technologies analytiques permettent à l'aide d'isotopes stables d'étudier directement le métabolisme et les fonctions des acides gras in vivo chez l'homme, jusqu'au niveau des récepteurs des neurotransmetteurs (N. Salem Jr).

Mais, jusqu'ici, les acides gras deutérés étaient utilisés sous forme libre, ce qui ne permettait pas d'emprunter les chemins préférentiels, les chemins ciblés. L'équipe de J.T. Brenna commence l'étude, chez des nouveau-nés primates, d'acides gras deutérés fixés en sn-2 sur un triglycéride et également en sn-2 sur divers phospholipides, l'acide gras non marqué dans les autres positions étant le palmitique. 
II s'avère d'ores et déjà que, en pourcentage de la dose, l'incorporation d'acide arachidonique venant de PL-AA est significativement plus grande dans le cerveau que celle venant de TG-AA, même si celle des TG-AAsn-2 est remarquable.

La voie pour après-demain s'ouvre.

Illustrations

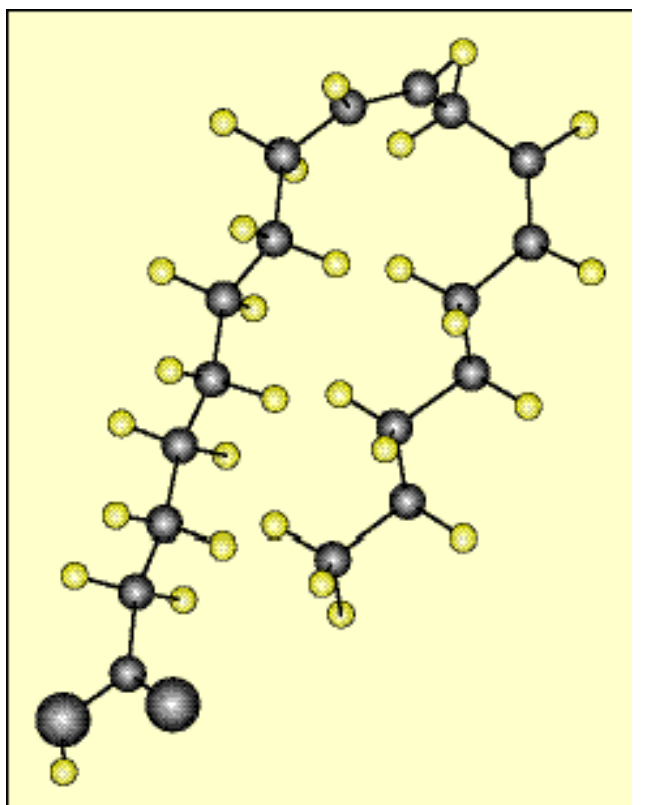

Figure 1. Acide linoléique dans l'eau (une des conformations possibles). Structure calculée par Clotilde Policar, Institut de chimie moléculaire d'Orsay. Laboratoire de chimie bio-organique \& chimie bio-inorganique, université de Paris Sud, 91405 Orsay Cedex. 


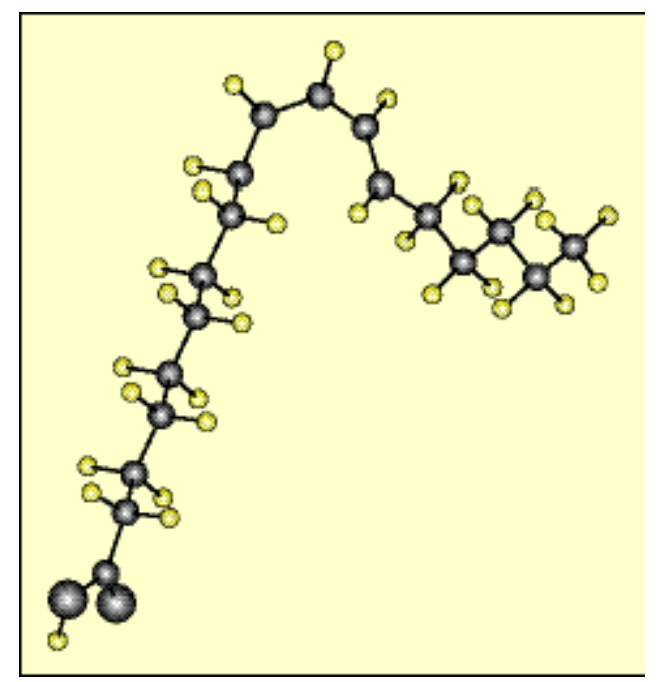

Figure 2. Acide ruménique (C18:2n-7t,9cis,11trans) dans l'eau (une des conformations possibles). Calcul Clotilde Policar.

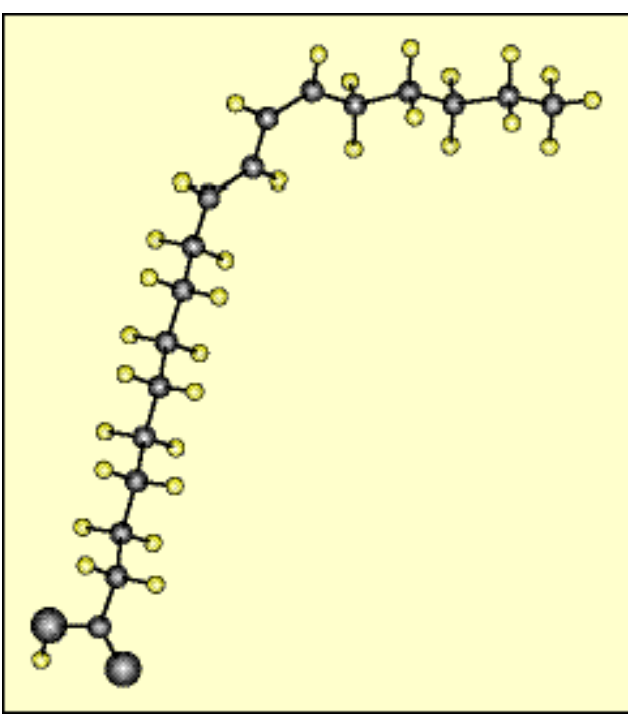

Figure 3. Isomère 10t12cCLA dans l'eau (une des conformations possibles). Calcul Clotilde Policar. 